\title{
Relationships between Galactic Radio Continuum and $\mathrm{H} \alpha$ Emission
}

\author{
L. E. Cram, A. J. Green and D. C.-J. Bock ${ }^{1}$ \\ School of Physics, University of Sydney, NSW 2006, Australia \\ L.Cram@physics.usyd.edu.au \\ 1 Present address: Radio Astronomy Laboratory, \\ University of California, Berkeley, CA 94720, USA \\ Received 1997 September 2, accepted 1997 December 22
}

\begin{abstract}
Radio continuum emission due to thermal bremsstrahlung and optical $\mathrm{H} \alpha$ spectral line emission arise from processes involving similar atomic entities and physical conditions. The relationship between the flux density of the emission from the two processes is mainly a function of the electron temperature of the emitting region, modified by other factors such as the mode of radiation transfer in the hydrogen spectrum. On the other hand, radio continuum radiation due to non-thermal synchrotron emission is formed by species and processes not involved in thermal emission. As a consequence, differences between the observed radio continuum emission and $\mathrm{H} \alpha$ emission from cosmic sources can provide reliable information on a variety of important physical aspects of the sources, including the relative importance of thermal and non-thermal radio emission and the degree of optical obscuration. This paper reviews the theory of the formation of $\mathrm{H} \alpha$ and the radio continuum in the interstellar medium (ISM), discusses some of the factors that must be considered in comparing observations made in the two frequency regimes, and summarises the properties of some classes of galactic object that emit both optical and radio radiation.
\end{abstract}

Keywords: radiation mechanisms: line, continuum — ISM: structure

\section{Introduction}

The proposed UKST H $\alpha$ Survey will detect Galactic optical emission in the $\mathrm{H} \alpha$ line, which is produced by electronic transitions from the $n=3$ to the $n=2$ bound level in atomic hydrogen. It will be of interest to compare this emission with Galactic thermal continuum radio emission, which is formed by thermal electrons moving in the presence of protons (hydrogen nuclei). In (low-density) interstellar thermal plasmas, the excitation of the $n=3$ level in hydrogen and the ionisation of atomic hydrogen are intimately connected, and so at first sight we would expect that radiation in $\mathrm{H} \alpha$ and in the thermal radio continuum would be quite similar.

However, there are many physical mechanisms and instrumental characteristics which conspire to produce marked differences in the appearance of cosmic sources in optical $\mathrm{H} \alpha$ radiation and in the radio continuum. This paper outlines the basic theory of the relevant emission processes and points out some of the effects that complicate the interpretation of observed similarities and differences in the intensity distribution of optical and radio emission from the Galactic interstellar medium.

Primary references to the topics covered here include the papers in the volume edited by Menzel (1962), and the books by Osterbrock (1974), Aller (1987), Longair (1994) and Kirk, Melrose \& Priest (1994).

\section{Basic Mechanisms of Thermal Emission}

In this section we summarise relevant radiation theory and present the relationship between $\mathrm{H} \alpha$ and radio-frequency radiation from a thermal hydrogen plasma.

An optically thin population of excited hydrogen atoms will radiate in the $\mathrm{H} \alpha$ spectral line a power $\left(\right.$ erg s $\mathrm{s}^{-1} \mathrm{sr}^{-1}$ or $10^{-7} \mathrm{~W} \mathrm{sr}^{-1}$ )

$$
j_{\alpha}=\frac{h \nu}{4 \pi} A_{32} N_{3}
$$

where $A_{32}$ is the Einstein coefficient for spontaneous emission and $N_{3}$ is the number density of hydrogen in the $n=3$ excited state. (Note that the rate of collisional transitions between the $L=S, P, D$ angular momentum states of $n=3$ may be too low to promote a state of detailed balance in low-density regions of the ISM, and that a more complete theory would need to consider these states separately.) In a plasma in statistical equilibrium (see Mihalas 1978), the population density $N_{3}$ is determined by the statistical balance between the various opportunities for radiative and collisional excitation and destruction of the state. The radiative rates depend on 'external' radiation, such as that from an exciting star, and in 'internal' radiation from the gas itself. The collisional rates are dominated by electron collisions, and so depend on the electron density and electron temperature. 
The population density $N_{3}$ can be related to the proton and electron densities, $N_{p}$ and $N_{e}$, by

$$
\begin{aligned}
N_{3}= & b_{3} \frac{g_{3}}{2 U_{0}(T)} \\
& \times\left(\frac{h^{2}}{2 \pi m k T}\right)^{\frac{3}{2}} e^{-\left(h \nu_{0}-\chi_{3}\right) / k T} N_{p} N_{e} .
\end{aligned}
$$

Here, $g_{3}$ is the statistical weight and $\chi_{3}$ the ionisation energy from $n=3, U_{0}$ is the partition function of atomic hydrogen, and $b_{3}$ is the departure coefficient reflecting the difference between the actual population of the level and the population that would occur in local thermodynamic equilibrium at the same temperature and the same values of $N_{e}$ and $N_{p}$.

Values of the departure coefficients may be predicted by solving the equations of statistical balance appropriate to various circumstances. In view of the complexity of the coupled equations, a number of convenient approximations are usually made in the analysis of low-density, radiatively excited nebulae ('Hı regions'). Perhaps the simplest approximation is that any photon produced in the gas is immediately radiated away. This approximationcalled 'Case A' - is not usually a valid approximation in cosmic plasmas. A slightly more complicated approximation which is often valid is the so-called 'Case B' recombination. In this approximation it is supposed that every Lyman line photon is scattered locally many times, ultimately being converted to Lyman- $\alpha$ and a higher-series photon, while all of the higher-series photons are emitted with no further scattering.

When Case B is valid, and the density is so low that collisional excitation and de-excitation can be ignored, it is straightforward to set up and solve a system of statistical balance equations for the ionisation equilibrium and bound-level population densities in hydrogen. These yield self-consistent values of the departure coefficient $b_{3}$ and other parameters. The value of $b_{3}$ depends on the electron temperature and the nature of the ambient radiation field, and might be typically $b_{3}=0 \cdot 1$. Under Case B conditions the ratio of the emissivity of any Balmer line to that in $\mathrm{H} \alpha$ (the Balmer decrement) is also readily predicted. Agreement or otherwise between the predicted and observed ratios then provides a check on the validity or otherwise of the assumption of Case B conditions. Alternatively, any difference between the predicted and observed ratio allows an estimate of the extinction if Case B is assumed.

The approximations underlying Case B equilibrium are not always satisfied in actual emission regions. A more complete and accurate approach to the spectroscopic diagnosis of $\mathrm{H} \alpha$ emission from the interstellar medium requires a computer study of a complex set of statistical balance equations. This approach is particularly important in regions with high densities (where collisions are relatively more important) and near dynamical features such as contact discontinuities, ionisation fronts and shock waves (where density gradients and time-dependent processes might be important).

A volume of cosmic plasma containing thermal electrons and protons will radiate thermal bremsstrahlung according to (e.g. Osterbrock 1974, Eq. 4.22)

$$
\begin{aligned}
j_{\nu}^{f f}= & \frac{1}{4 \pi} \frac{32 e^{4} h}{3 m^{2} c^{3}}\left(\frac{\pi h \nu_{0}}{3 k T}\right)^{\frac{1}{2}} \\
& \times e^{-h \nu / k T} g_{f f}(\nu, T) N_{i} N_{e} .
\end{aligned}
$$

Here, $g_{f f}$ is the Gaunt factor for free-free radiation, for which an approximation is available (e.g. Rohlfs 1986, Eq. 8.27). The total brightness of thermal radiation along a specified line of sight is the integral of this expression, provided that the line of sight is optically thin. For sufficiently low frequencies and sufficiently high emission measures (the emission measure is the integral $E M=\int N_{e} N_{p} d s$ along the line of sight), the line of sight will not be optically thin, and the brightness of the radio thermal continuum is then given by the (black-body) Planck function. In this case, the shape of the radio continuum spectrum as it varies from the optically thick (low-frequency) to the optically thin (high-frequency) part provides additional information on physical conditions in the plasma (e.g. Rohlfs 1986, Section 8-4).

The consequence of the theory outlined above is the prediction of a close relationship between the thermal radio continuum flux density and $\mathrm{H} \alpha$ intensity along a line of sight free from other emission or any absorption processes. The relationship corresponds to

$$
\frac{j_{\alpha}}{j_{\nu}^{f f}}=C_{I} \frac{b_{3}}{U_{0}(T) g_{f f}(\nu, T)} \frac{e^{\chi_{3} / k T}}{T}
$$

where $C_{I}$ depends only on physical and atomic constants. Note that in this equation the factor $b_{3} / U_{0} g_{f f}$ is of order $0 \cdot 1$, but would be uncertain by perhaps as much as an order of magnitude as a result of uncertainties in the effects of collisions and radiative transfer in the determination of the departure coefficient.

\section{Observational Considerations}

Three major effects ensure that the ratio derived above does not usually correspond to the observed ratio of the flux density in the radio continuum to the brightness of $\mathrm{H} \alpha$ : (a) the extinction of $\mathrm{H} \alpha$ emission by grains, (b) the contribution of nonthermal radio-frequency radiation to the detected radio flux density, and (c) the differences between the 
responses of optical and radio telescopes to extended sources, most importantly the failure of synthesis radio telescopes to detect structural components with large angular scales.

The general extinction of the Galaxy follows a relationship of the form $A_{v}=0 \cdot 15(\operatorname{cosec}|b|-1)$, where $b$ is the Galactic latitude. This implies that there is at least 1 magnitude of extinction (in $V)$ if $|b|<7 \cdot 5^{\circ}$. In addition, there can be local enhancements of extinction in star-forming regions, which are often associated with $\mathrm{H} \alpha$ emission regions. The extinction implies that the observed $\mathrm{H} \alpha$ flux from many emitting regions, especially the more distant ones, is severely attenuated. Although there are problems as noted above, it is possible to attempt to correct for this attenuation using observations of the 'Balmer decrement' (the ratios of fluxes in the lines of the Balmer series), provided that (1) the attenuation is not too great, (2) the frequency dependence of the grain attenuation is known, and (3) the intrinsic ratio of the Balmer line fluxes can be reliably predicted.

Non-thermal radio radiation from the Galaxy is due to synchrotron emission from relativistic electrons. The synchrotron emissivity from an electron population with an energy distribution $N(E) d E=K E^{-\gamma} d E$ per unit volume is given by (e.g. Ginzberg \& Syrovatskii 1965)

$$
\begin{aligned}
j_{\nu}^{n t}= & \alpha(\gamma) \frac{e^{3}}{m c^{2}}\left(\frac{3 e}{4 \pi m^{3} c^{5}}\right)^{\frac{\gamma-1}{2}} \\
& \times H_{\perp}^{\frac{\gamma+1}{2}} K \nu^{-\frac{\gamma-1}{2}} .
\end{aligned}
$$

Here $\alpha(\gamma)$ is a dimensionless constant of order unity, and $H_{\perp}$ is the component of the magnetic field perpendicular to the line of sight. The power-law index $\gamma \approx 2$ characterises much of the Galactic relativistic electron population in the energy range responsible for synchrotron emission in the radio continuum, so that the spectrum of the synchrotron radiation varies as $\sim \nu^{-1 / 2}$. Consequently, the spectrum of the radio continuum can distinguish thermal and non-thermal radiation, since thermal radiation is either approximately flat $\left(\sim \nu^{0}\right)$ in optically thin regions, or varying as $\nu^{2}$ in optically thick regions. Another distinction between thermal and non-thermal radio continuum radiation is that, provided that the magnetic field of the source region is sufficiently ordered, and that depolarising effects are small along the line of sight, synchrotron radiation is polarised while thermal Bremsstrahlung is not.

To a first approximation Galactic radio continuum radiation consists of two non-thermal components and two thermal components. The two main non-thermal components are: (1) a disk of emission coinciding with the plane of the Galaxy, which produces an all-sky brightness peaking towards $|b|=0$ and $\ell=0$, and (2) localised emission from supernova remnants dotted throughout the Galaxy, but concentrated towards the plane and towards the inner Galaxy. The former component arises from diffuse cosmic ray electrons and the general Galactic magnetic field, while the latter arises from electrons accelerated, and magnetic fields disturbed, by the supernova. The two main thermal components are: (1) diffuse emission highly concentrated towards the plane and the centre, and (2) discrete sources associated with HII regions of various types. The former component presumably arises in the diffuse warm ISM, while the latter is predominantly associated with star-forming regions.

In view of the $\nu^{-1 / 2}$ spectrum of non-thermal radiation, and the flat spectrum of optically thin thermal radiation, the ratio of thermal to nonthermal radio continuum emission tends to increase with increasing radio frequency. At 'low' radio frequencies $(\sim 1 \mathrm{GHz})$ non-thermal emission dominates the diffuse Galactic radiation field, while at 'high' radio frequencies $(\sim 10 \mathrm{GHz})$ the diffuse thermal component tends to dominate (Handa et al. 1987). The task of distinguishing between thermal and non-thermal discrete sources (i.e. between HII regions and supernova remnants in the simplest case) is often straightforward, but in many complex regions the separation is very difficult and frustrates attempts to decipher the observed structures in terms of evolutionary trends, triggering mechanisms and physical relationships. Additional observational material, such as the presence of shock-excited $\mathrm{OH}$ maser emission, can help elucidate the relationships between thermal and non-thermal processes (e.g. Frail et al. 1996).

The role of the telescope in 'filtering' $\mathrm{H} \alpha$ or radio continuum radiation can be crucial. For optical observations made with an $\mathrm{H} \alpha$ filter (either digitally or photographically), the telescope will respond to all angular scales in the field that are larger than the angular resolution, although the radiation will be detected only where it rises above the system noise (including the general emission of the night sky). Optical spectroscopy (or Fabry-Perot interferometry) can better distinguish $\mathrm{H} \alpha$ against the sky background and can also provide kinematic data from the Doppler shift of the line.

Radio-frequency images made by scanning with a single dish are analogous in many respects to optical observations made through a filter, although the angular resolution of the largest single-dish radio telescopes (10 arcmin for a $100 \mathrm{~m}$ telescope at 1 $\mathrm{GHz}$ ) is far poorer than that of quite modest optical telescopes with sufficient collecting power to attain adequate signal-to-noise ratios in $\mathrm{H} \alpha$ (e.g. 0.5 arcsec for a $0 \cdot 25 \mathrm{~m}$ telescope). 

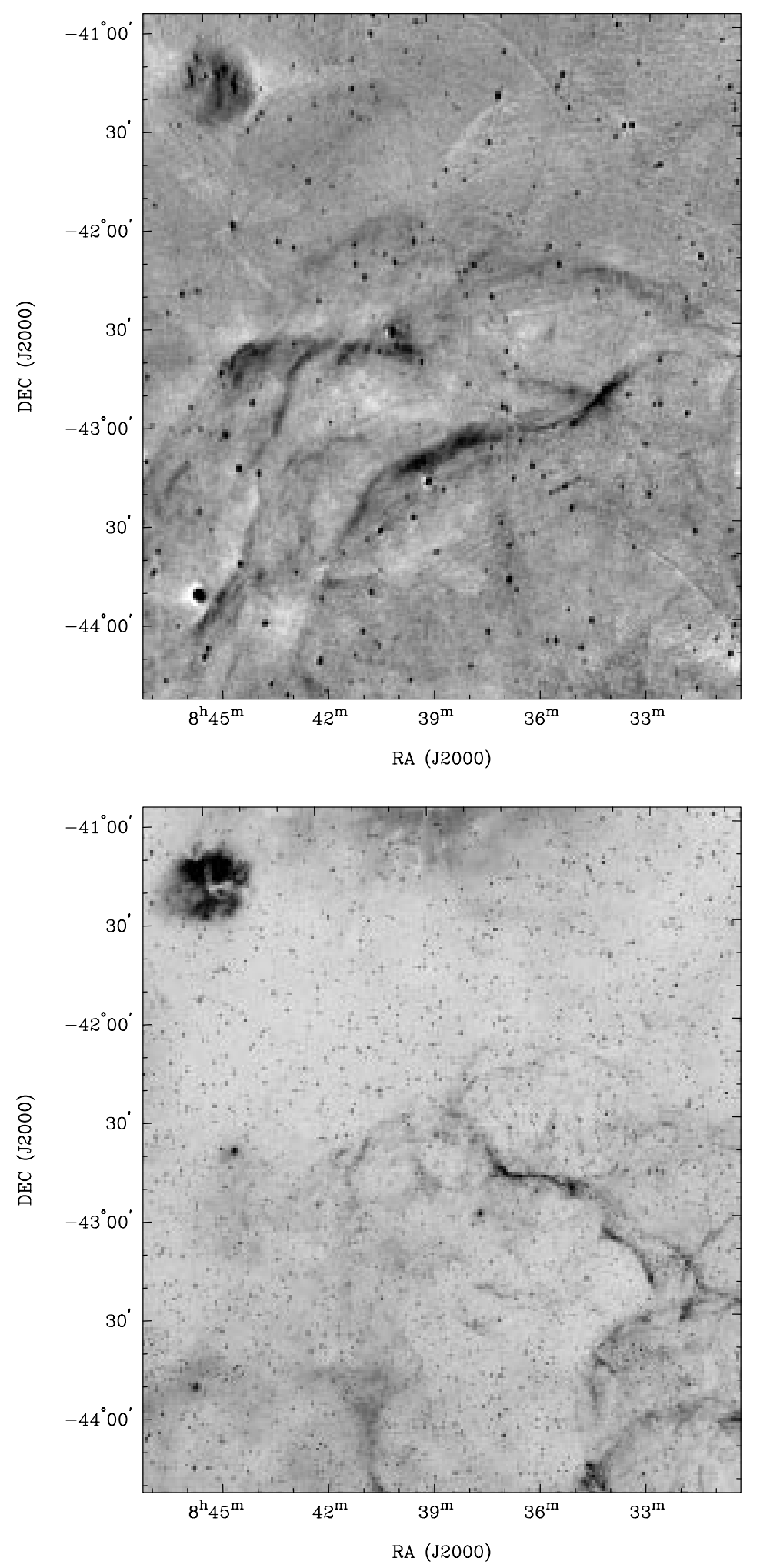

Figure 1-Comparison between a radio continuum image made with the MOST at $843 \mathrm{MHz}$ (top) and an $\mathrm{H} \alpha$ image of the same region of the Vela SNR (bottom). The object in the top left of the image is an HII region. The radio image is from Bock (1997), and the H $\alpha$ image has been kindly provided by Dr M. Bessell.

The parallels between optical and radio imaging are less direct for observations made with a radio interferometer. It is true that an interferometer, like a single-dish telescope, possesses a lower limit to the angular scales it can resolve in the image.
This correspond to the largest spacings of the interferometer. Interferometers generally have longer maximum spacings than those of single dishes, so interferometer images usually have higher angular resolution. However, in comparing optical (or 


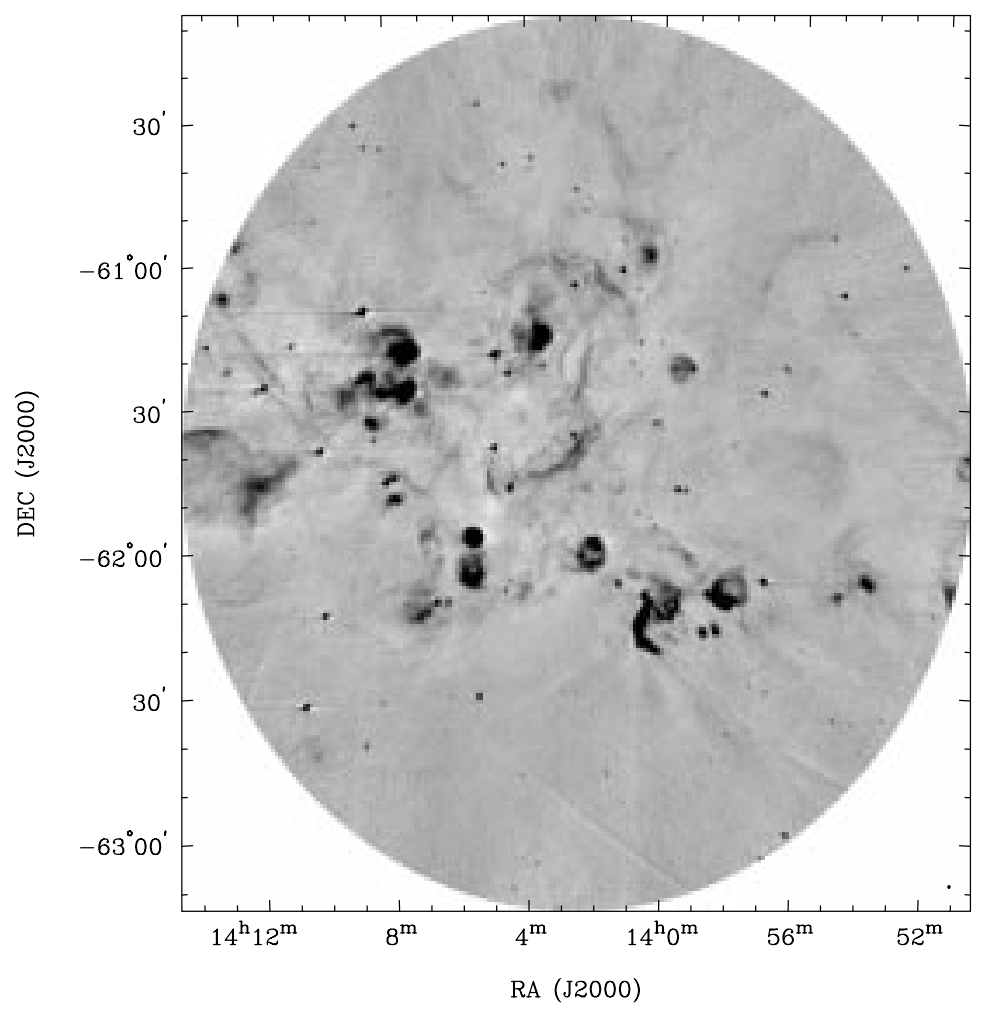

Figure 2-An image in the radio continuum at $843 \mathrm{MHz}$, made as part of the new MOST survey of the Galactic Plane. The small dot to the lower right indicates the size of the MOST beam.

single-dish radio) images and images made with interferometers, it is important to recognise that the normal mode of operation of an interferometer does not measure visibilities on spacings smaller than the distance between the closest elements. This has the effect of forming a spatially high-pass filtered image with zero mean, in which source structure on large angular scales is not detected. The resulting image can be quite misleading unless this fact is recognised.

In particular, the general diffuse non-thermal radiation of the Galactic plane, which has a brightness far greater than much of the small-scale emission from HII regions and supernova remnants, is simply not detected by most interferometers. Thus, although the ridge of radio continuum emission lying towards the Galactic centre has a total flux density of well over $1000 \mathrm{Jy}$ at $1 \cdot 4 \mathrm{GHz}$ (in $|b|<1 \cdot 5,-5<\ell<5$ ), the total flux density in 'resolved' objects whose VLA images have been published (i.e. Sgr A, B, C, $\mathrm{D}, \mathrm{E}$ and other complexes) is much less than $100 \mathrm{Jy}$ (cf. Handa et al. 1987; Salter \& Brown 1988; Liszt 1988). Comparisons between radio interferometer and optical images must be made with particular attention to the absence of low angular scales in the former.

\section{Prospects for Multi-wavelength Studies of the ISM}

The theory outlined above pertains to emission mechanisms per se, and does not touch on the broader question of the physical mechanisms responsible for the structure that is seen in $\mathrm{H} \alpha$ and the radio continuum. Reviews of these mechanisms can be found in texts (e.g. Longair 1992; Lozinskaya 1992; Kirk, Melrose \& Priest 1994) and conference proceedings (e.g. Hollenbach \& Thronson 1987). It is clear from this literature that there are several outstanding problems for which combined $\mathrm{H} \alpha$ and radio continuum observations would provide importance guidance to theory.

One example illustrating this point concerns the correlation of fine-scale $\mathrm{H} \alpha$ structure and radio continuum structure in supernova remnants. In some remnants (e.g. HB3, see Fesen et al. 1995; IC443, see Duin \& van der Laan 1975) there are large areas with a close correspondence between the shape and brightness distributions in the two forms of emission, suggesting that non-thermal electrons are accelerated and/or the magnetic field intensified in close proximity to the site of thermal excitation of $\mathrm{H} \alpha$. In other remnants (e.g. the Cygnus loop, Lozinskaya 1992, especially pp. 134-143) there is close correlation in some of the fine structure but also uncorrelated structures, suggesting a diversity of excitation mechanisms in different parts of the remnant. In yet other remnants, such as the Vela complex, there is only a small degree of correlation. The situation in Vela is illustrated in Figure 1, which compares an image made using the Molonglo Observatory Synthesis Telescope (MOST) in the radio continuum at $843 \mathrm{MHz}$ with an $\mathrm{H} \alpha$ image 
made by Dr M. Bessell of Mount Stromlo Observatory using a large-format CCD detector mounted behind a $0.2 \mathrm{~nm} \mathrm{H} \alpha$ filter and a small camera attached to the MSSSO $0.4 \mathrm{~m}$ telescope.

It seems likely that a basic factor in the coexistence or otherwise of $\mathrm{H} \alpha$ and radio continuum fine structure is the nature of the pre-existing interstellar medium: if mechanical energy from the supernova blast propagates into a low-density, hot ISM phase then little $\mathrm{H} \alpha$ radiation would be expected, while at the same time structuring of the non-thermal radio continuum could arise through waves, instabilities or inhomogeneities in the density or magnetic field. On the other hand, propagation into a dense, cool region would lead to $\mathrm{H} \alpha$ radiation from regions lying in the appropriate range of density and temperature. The question of associated non-thermal emission from such high-density regions requires further research, for the available models of particle acceleration rest on the theory of collisionless shocks. Such shocks might exist close to the collisional shocks responsible for the $\mathrm{H} \alpha$ emission, but are unlikely to be precisely coincident.

The MOST has completed a survey of the radio continuum emission from the southern Galactic plane $\left(|b|<1 \cdot 5^{\circ},-5^{\circ}<\ell<240^{\circ}\right)$ with an angular resolution of 40 arcsec and a sensitivity of about 5 mJy per beam area (Whiteoak et al. 1989; Whiteoak \& Green 1996; Green \& Cram 1998). This survey is ideally suited to extensive studies of the correlation between the radio continuum and optical $\mathrm{H} \alpha$ radiation that will become possible when the UKST H $\alpha$ survey is under way. In addition to the existing MOST survey, a second survey has commenced, exploiting the new wide-field capability of the MOST (Bock 1997; Bock, Large \& Sadler 1998), and this will provide additional high-resolution, low-noise panoramic radio continuum images of the southern Galactic plane. Figure 2, one of the first Galactic images made in the new MOST survey, illustrates the coverage and sensitivity of single images in the survey to fine-scale continuum emission, and suggests that comparisons between $\mathrm{UKST} \mathrm{H} \alpha$ images and radio continuum images are likely to be a fruitful way to explore the Galactic ISM.

There are clearly excellent prospects for significant progress on a number of outstanding problems in Galactic astrophysics that will emerge from multiwavelength studies of the interstellar medium which include both $\mathrm{H} \alpha$ and radio continuum imaging.

Aller, L. H. 1987, Physics of Thermal Gaseous Nebulae (Dordrecht: Reidel)

Bock, D. C.-J. 1997, Wide Field Aperture Synthesis Radio Astronomy, PhD thesis, University of Sydney

Bock, D. C.-J., Large, M. I., \& Sadler, E. M. 1998, (in preparation)

Duin, R. M., \& van der Laan, H. 1975, A\&A, 40, 111

Fesen, R. A., Downes, R. A., Wallace, D., \& Normandeau, M. 1995, AJ, 110, 2876

Ginzberg, V. L., \& Syrovatskii, S. I. 1965, ARA\&A, 3, 297

Green, A. J., \& Cram, L. E. 1998 (in preparation)

Handa, T., Sofue, Y., Nakai, N., Hirabayashi, H., \& Inoue, M. 1987, PASJ, 39, 709

Hollenbach, D. J., \& Thronson, H. A. (eds) 1987, Interstellar Processes (Dordrecht: Reidel)

Kirk, J. G., Melrose, D. B., \& Priest, E. R. 1994, Plasma Astrophysics (Berlin: Springer)

Liszt, H. 1988, in Galactic and Extragalactic Radio Astronomy, eds G. L. Vershuur \& K. I. Kellermann (Berlin: Springer), p. 359

Longair, M. S. 1994, High Energy Astrophysics (two volumes) (Cambridge Univ. Press)

Lozinskaya, T. A. 1992, Supernovae and Stellar Winds in the Interstellar Medium (Chicago: AIP)

Menzel, D. H. (ed.) 1962, Selected Papers on Physical Processes in Ionised Plasmas (New York: Dover)

Mihalas, D. 1978, Stellar Atmospheres (2nd edn) (San Francisco: W. H. Freeman)

Osterbrock, D. E. 1974, Astrophysics of Gaseous Nebulae (San Francisco: W. H. Freeman)

Rohlfs, K. 1986, Tools of Radio Astronomy (Berlin: Springer)

Salter, C. J., \& Brown, R. L. 1988, in Galactic and Extragalactic Radio Astronomy, eds G. L. Vershuur \& K. I. Kellermann (Berlin: Springer), p. 1

Whiteoak, J. B. Z., \& Green, A. J. 1996, A\&A Suppl., 118, 329

Whiteoak, J. B. Z., Large, M. I., Cram, L. E., \& Piestrzynski, B. 1989, PASA, 8,176 